\title{
Evidence for RPGRIP1 gene as risk factor for primary open angle glaucoma
}

\author{
Lorena Fernández-Martínez ${ }^{1}$, Stef Letteboer ${ }^{2}$, Christian Y Mardin ${ }^{3}$, Nicole Weisschuh ${ }^{4}$, Eugen Gramer ${ }^{5}$, \\ Bernhard HF Weber ${ }^{6}$, Bernd Rautenstrauss ${ }^{1,8}$, Paulo A Ferreira ${ }^{7}$, Friedrich E Kruse ${ }^{3}$, André Reis ${ }^{1}$, \\ Ronald Roepman ${ }^{2}$ and Francesca Pasutto*,1
}

Glaucoma is a genetically heterogeneous disorder and is the second cause of blindness worldwide owing to the progressive degeneration of retinal ganglion neurons. Very few genes causing glaucoma were identified to this date. In this study, we screened 10 candidate genes of glaucoma between the D14S261 and D14S121 markers of chromosome 14q11, a critical region previously linked to primary open-angle glaucoma (POAG). Mutation analyses of two large cohorts of patients with POAG, normal tension glaucoma (NTG) and juvenile open-angle glaucoma (JOAG), and control subjects, found only association of non-synonymous heterozygous variants of the retinitis pigmentosa GTPase regulator-interacting protein 1 (RPGRIP1) with POAG, NTG and JOAG. The 20 non-synonymous variants identified in RPGRIP1 were all distinct from variants causing photoreceptor dystrophies and were found throughout all but one domain (RPGR-interacting domain) of RPGRIP1. Among them, 14 missense variants clustered within or around the C2 domains of RPGRIP1. Yeast two-hybrid analyses of a subset of the missense mutations within the C2 domains of RPGRIP1 shows that five of them (p.R598Q, p.A635G, p.T806I, p.A837G and p.I838V) decrease the association of the C2 domains with nephrocystin-4 (NPHPH). When considering only these five confirmed C2domain mutations, the association remains statistically significant $(P=0.001)$. Altogether, the data support that heterozygous non-synonymous variants of RPGRIP1 may cause or increase the susceptibility to various forms of glaucoma and that among other factors, physical impairment of the interaction of RPGRIP1with different proteins may contribute to the pathogenesis of forms of glaucoma.

European Journal of Human Genetics (2011) 19, 445-451; doi:10.1038/ejhg.2010.217; published online 12 January 2011

Keywords: glaucoma; RPGRIP1; mutation screening

\section{INTRODUCTION}

Glaucoma is the second most prevalent cause of blindness worldwide and comprises a heterogeneous group of complex neurodegenerative diseases of the retina. ${ }^{1,2}$ Of the different forms of glaucoma, primary open-angle glaucoma (POAG, OMIM 137760) is the most common affecting $>35$ million people worldwide. ${ }^{3}$ POAG is characterized by primary loss of retinal ganglion cells along with supporting glia and vasculature, specific atrophy of the optic nerve, progressive loss of neuroretinal rim of the optic disk, with corresponding progressive, peripheral visual field loss and, if untreated, eventual blindness. Elevated intraocular pressure (IOP) seems to be a major risk factor, although a number of patients develop glaucoma also in the absence of elevated IOP. ${ }^{4}$ In this case, glaucoma is defined as normal tension glaucoma (NTG). On the basis of age of onset before 40, a juvenile form of open angle glaucoma (JOAG) is differentiated from adult-onset POAG. ${ }^{5}$ A family history of POAG is a well-established risk factor, indicating that specific genetic variants may contribute to pathogenesis, and a multifactorial etiology is suggested. ${ }^{6}$ In some large pedigrees, though, glaucoma segregates as a Mendelian trait with reduced penetrance and variable expressivity, but most cases are sporadic.
Currently, through linkage studies, 14 chromosomal loci for POAG (GLC1A-N) have been identified (HUGO, http://www.genenames.org/ index.html) and many more have been reported in the literature. ${ }^{7}$ However, only three causative genes have been described so far, including: myocilin (MYOC/GLC1A) ${ }^{8}$ optineurin $(\text { OPTN/GLC1E })^{9}$ and WD repeat domain 36 (WDR36/GLC1G).${ }^{10}$ Altogether, mutations in these genes account for $<10 \%$ of POAG cases. ${ }^{11}$ In addition, association studies have implicated at least 20 other genes, ${ }^{7,12}$ among them cytochrome P450-1B (CYP1B1) ${ }^{13}$ and neurotrophin 4 (NTF4), ${ }^{14}$ contributing to the pathogenesis of glaucoma, ${ }^{7}$ but these are still insufficient to explain the most significant fraction of POAG cases. ${ }^{15}$ This may not come as a surprise, as the POAG phenotype itself seems complex and to result from diverse pathological processes that involve, but are not limited to, the retina and optic nerve, the aqueous humor outflow pathways and even, as suggested recently, the cerebrospinal fluid dynamics. ${ }^{16}$

In 2000, Wiggs et al ${ }^{17}$ published a first genome-wide linkage scan involving an initial set of 41 multiplex families and a second one of 33, where several loci, including regions on chromosome 2, 14, 17 and 19, were linked to POAG. We, therefore, focused our efforts on

${ }^{1}$ Institute of Human Genetics, Friedrich-Alexander University of Erlangen-Nuremberg, Erlangen, Germany; ${ }^{2}$ Department of Human Genetics, Radboud University Nijmegen Medical Centre, Nijmegen, The Netherlands; ${ }^{3}$ Department of Ophthalmology, University Erlangen-Nuremberg, Erlangen, Germany; ${ }^{4}$ Molecular Genetics Laboratory, University Eye Hospital, Tübingen, Germany; ${ }^{5}$ University Eye Hospital, Würzburg, Germany; ${ }^{6}$ Institute of Human Genetics, University Regensburg, Regensburg, Germany; ${ }^{7}$ Department of Ophthalmology, Duke University Medical Center, Durham, NC, USA

*Correspondence: Dr F Pasutto, Institute of Human Genetics, Friedrich-Alexander-University of Erlangen-Nuremberg, Schwabachanlage 10, Erlangen 91054, Germany.

Tel: +49 9131 8522055; Fax: +49 9131 8523232; E-mail: francesca.pasutto@uk-erlangen.de

8Present address: MGZ-Medical Genetics Centre, 80335 Munich, Germany.

Received 19 August 2010; revised 29 October 2010; accepted 10 November 2010; published online 12 January 2011 
chromosomal region $14 \mathrm{q} 11$. A further genome-wide scan comprising 1327 individuals and 146 families of the Barbados family study, provided evidence for linkage to chromosomes 1, 2, 9 and 11, and also gave weak support for the existence of a locus on chromosome $14{ }^{18}$ To our knowledge, follow-up studies to refine the genetic intervals and to assess possible candidate genes located in these regions have been not yet reported.

We screened the candidate chromosomal region, covering $6.3 \mathrm{Mb}$ between markers D14S261 and D14S121, for putative functional candidate genes. Among more than 266 known and predicted genes annotated in the critical region, we selected 10 genes for mutational analysis, because among other factors of their expression pattern in eyes tissues, especially in retina. These 10 genes were: adenylate cyclase type IV (ADCY4), B-cell/lymphoma 2-like 2 (BCL2L2), defender against cell death 1 (DAD1), interferon-stimulated transcription factor 3- $\gamma$ (ISGF3G), matrix metalloproteinase 14 (MMP14), neural retina leucine zipper $(N R L)$, oxidase cytochrome $c$ assembly 1-like (OXA1L), salivary protein-like 2 (SALL2), zinc-finger protein 219 (ZNF219) and retinitis pigmentosa GTPase regulator-interacting protein 1 (RPGRIP1).

In this work, we report the results of the mutation analysis of the 10 candidate genes in a small explorative collective of POAG patients and importantly, the mutation screen of the RPGRIP1 gene in two different cohorts of patients with POAG, NTG and JOAG from Germany and their respective control groups. We have found association of non-synonymous variants predicted to alter the sequence of known structural and functional domains of RPGRIP1in patients with POAG, NTG and JOAG.

\section{METHODS}

\section{Study population}

The study was approved by the ethics review board of the Medical Faculty of the University of Erlangen-Nuremberg (Germany) and was in accordance with the tenets of the Declaration of Helsinki. All subjects gave informed consent before entering the study.

The first group of patients with glaucoma consisted of 399 subjects of German (European) origin: 270 had high-pressure POAG, 47 had JOAG, and 82 had NTG. The 376 control subjects were all of German origin and were recruited from the same geographic region as the patients. All individuals underwent standardized clinical examinations for glaucoma at the Ophthalmological Department of the University of Erlangen-Nuremberg, Erlangen. Further recruitment and clinical data have been previously reported. ${ }^{13,14,19}$ All subjects were also screened for MYOC, OPTN (data not shown), WDR36, ${ }^{19}$ $C Y P 1 B 1^{13}$ and $N T F 4^{14}$ mutations (to note that patients with mutations in one of these candidate genes were also included in the present screening study as the etiology of glaucoma is still unknown).

The second group comprised 383 unrelated patients (304 NTG and 79 POAG) and 104 unrelated control subjects of German descent. The subjects of these groups were clinically investigated at the University Eye Hospital in Würzburg and Tübingen with the same clinical procedure as in Erlangen. Clinical details have been reported elsewhere. ${ }^{20}$

Altogether, the age of patients range from 14 to 96 with a mean of $66.5 \pm 14.1$ years, $61 \%$ were females and $37 \%$ had a family history of glaucoma. The age of controls subjects range from 34 to 97 with a mean of $72.1 \pm 6.9$ years. A total of $59.8 \%$ of control subjects were females.

\section{Mutational screening}

Genomic DNA was extracted from peripheral blood leukocytes with automated techniques (AutoGenFlex 3000; Autogen, Holliston, MA, USA) using DNA chemistry (Flexigene; Qiagen, Hilden, Germany). Individual coding exons of the RPGRIP1 gene including flanking intronic and untranslated region sequences were amplified by PCR. Primer sequences were selected with Primer3 software (http://frodo.wi.mit.edu/cgi-bin/primer3/primer3_www.cgi/) and are available on request. Purified PCR products (AMPur; Agencourt Bioscience,
Beverly MA, USA, purified on a Biomek NX96 platform; Beckman Instruments, Fullerton, CA, USA) were sequenced using dye termination chemistry (Prism Fluorescent Dye Termination; ABI, Darmstadt, Germany). Purified sequence reactions (CleanSEQ; Agencourt Bioscience) were resolved on a capillary automated sequencer (3730 Sequence Analyzer; ABI) and analyzed with genome assembly software (Seqman software; DNAStar, Madison, WI, USA). Each variant was confirmed by a second independent analysis.

GenBank accession NM_020366 was used as cDNA reference sequence, NT_026437 as genomic reference sequence and NP_065099 as the reference protein sequence for RPGRIP1 (http://www.ncbi.nlm.nih.gov National Center for Biotechnology Information, Bethesda, MD, USA). Evolutionary conservation of non-synonymous variants was investigated with protein sequence alignment generated by ClustalW (http://www.ebi.ac.uk/clustalw/ European Molecular Biology Laboratory, Heidelberg, Germany) and compared with that presented by the Ensembl Database (http://www.ensembl.org).

\section{DNA constructs}

Gateway-adapted expression constructs were created using the Gateway cloning system (Invitrogen, Karlsruhe, Germany), and expression vectors used were described in Roepman et al. ${ }^{21}$ All PCR-generated fragments were verified by nucleotide sequencing. Mutations were introduced using the QuickChange site-directed mutagenesis kit (Stratagene, Amsterdam, Denmark).

\section{Analysis of interactions in yeast}

The Saccharomyces cerevisiae strain PJ69-4A7 $\alpha$ harboring LacZ and HIS3 reporter genes was used to evaluate the interaction of the wild-type and mutant C2 domains of RPGRIP1with nephrocystin-4 (NPHP4) in the yeast two-hybrid assay (Clontech, Saint-Germain-en-Laye, France). ${ }^{21}$ The transformants were selected by growth on SD medium lacking Trp at $30^{\circ} \mathrm{C}$ for 3-4 days. To test the expression of the LacZ gene, a colony lift filter assay was performed using X-GAL as a substrate. The transformants were also grown in SD medium minus His and minus Trp. The activities of reporters were analyzed in four independent transformants.

The interactions of the wild-type and mutant RPGRIP1 and NPHP4 proteins were quantified by determining the $\beta$-galactosidase activity using the Yeast $\beta$-Galactosidase Assay Kit (Pierce, Bonn, Germany) according to the manufacturer's instructions.

\section{Coimmunoprecipitation assays}

Constructs pcDNA3-HA-N4 4 L/EXP (N-terminal hemagglutinin (HA) tag) and p3xFlag-RPGRIP1 ${ }^{\mathrm{C} 2}-\mathrm{N}+\mathrm{C} 2-\mathrm{C} / \mathrm{EXP}$ ( $\mathrm{N}$-terminal $3 \times$ Flag tag) were used to express epitope-tagged proteins for coimmunoprecipitation. As control plasmid, p3xFlag-LRRK2 ${ }^{\mathrm{LRR}} / \mathrm{EXP}$ was used. COS- 1 cells were cotransfected with plasmid DNA using Lipofectamine 2000 (Invitrogen). Proteins were expressed for $24 \mathrm{~h}$, and cells were subsequently washed in PBS and lysed in ice-cold lysis buffer (50 mu Tris-HCl pH 7.5, $150 \mathrm{~mm} \mathrm{NaCl}, 0.5 \%$ Triton X-100 and complete protease inhibitor cocktail (Roche, Mannheim, Germany). Lysates were cleared by centrifugation at $4^{\circ} \mathrm{C}$ for $10 \mathrm{~min}$ at $14000 \mathrm{~g}$. Immunoprecipitation was performed with anti-HA monoclonal mouse antibody (Sigma, Taufkirchen, Germany) overnight at $4{ }^{\circ} \mathrm{C}$ accomplished through interaction with protein $\mathrm{A} / \mathrm{G}$ beads (Santa Cruz Biotechnology, New York, NY, USA). Beads were then washed four times with lysis buffer, mix with LDS-NuPAGE sample buffer (Invitrogen) and heated at $70^{\circ} \mathrm{C}$ for $10 \mathrm{~min}$. The immunocomplexes were then analyzed with SDS-PAGE followed by western blot analysis using anti-Flag monoclonal mouse antibody (clone M2, Sigma) and anti-HA antibody. Proteins were detected by chemoluminiscence (Amersham, Uppsala, Sweden).

\section{RESULTS}

\section{Mutational analysis}

Sequence analysis of the coding regions of the 10 selected positional and functional candidate genes (ADCY4, BCL2L2, DAD1, ISGF3G, MMP14, NRL, OXA1L, SALL2, ZNF219 and RPGRIP1) was performed in an explorative subset of 46 German patients with POAG chosen from the first group of patients and 46 control subjects. Overall 37 coding variants were found, 10 of which coded for non-synonymous 
amino acids. These 10 variants were further characterized, analyzing their presence in healthy subjects and their evolutionary conservation among different mammalian species (human, mouse, rat, cow and chimpanzee). Only three of these missense mutations, all located in RPGRIP1 gene, were both not present in healthy subjects and affecting evolutionary conserved amino acids. In line with these findings, RPGRIP1 arose as a potential promising candidate for POAG. Thus, we extended the screening for mutations in RPGRIP1 to the entire first group of patients and control subjects.

The systematic mutation analysis in RPGRIP1 led to the identification of 14 amino acid substitutions (Table 1 and Figure 1). Altogether, the amino acid changes accounted for $6.5 \%$ of the population of patients (26/399). A total of eight changes were detected in 376 healthy control subjects, accounting for only $2.1 \%(8 / 376)$ of the control group $(P=0.003$, two-tailed Fischer's exact test; $\mathrm{OR}=2.8,95 \%$ $\mathrm{CI}=1.4-5.5)$.

As most of the patients carried a missense mutation located in the C2 domains of RPGRIP1 (16 patients (4\%) against 3 control subjects $(0.8 \%), P=0.004, \mathrm{OR}=5.2,95 \% \mathrm{CI}=1.5-18$, Table 1 and Figure 1$)$, we screened the complete coding region of the RPGRIP1 C2 domains in a further German cohort of 383 glaucoma patients (304 NTG and 79 POAG) and 104 control subjects to support the observed association data in these domains. We could identify six novel amino acid substitutions in nine patients $(2.3 \%)$ and in two control subjects
(1.9\%; Table 1 and Figure 1). Altogether, the distribution of RPGRIP1 C2 domain variants found remained statistically significant between patients and controls despite the relatively small number of control individuals in the second studied cohort $(P=0.013$, two-tailed Fischer's exact test; $\mathrm{OR}=2.5,95 \% \mathrm{CI}=1.2-5.3$, Table 1 ).

In addition, $\mathrm{C} 2$ domain missense variants resulted to be more or less common among the different phenotype groups, if we take into account the small number of JOAG patients analyzed: $3.1 \%$ in POAG, $2.9 \%$ in NTG and $6.3 \%$ in JOAG. Moreover, these missense variants are almost equally distributed between the patients with high IOP (3.5\%, combined POAG and JOAG) and patients with normal IOP value $(2.9 \%, \mathrm{NTG})$.

The group of patients carrying RPGRIP1 missense mutations comprised both late juvenile and adult onset POAG at age of recruitment varying from 20 to 81 years (Table 2). Among these 35 identified patients, 22 had elevated maximum IOP ranging from 22 to $40 \mathrm{~mm} \mathrm{Hg}$, whereas 13 had pressure measurements in the normal range. In addition, two of these patients presented missense mutations also in MYOC and CYP1B1 genes (Table 2).

\section{RPGRIP1 yeast interaction and coimmunoprecipitation assays}

NPHP4 is one of the currently known partners of the C2 domains of RPGRIP1. ${ }^{21}$ To compare the functional relevance of the nonsynonymous variants found only in glaucoma patients with those

Table 1 RPGRIP1 sequence variants found in patients and control individuals

\begin{tabular}{|c|c|c|c|c|c|c|}
\hline \multirow[b]{2}{*}{ Nucleotide alteration } & \multirow[b]{2}{*}{$\begin{array}{l}\text { Amino acid } \\
\text { change }\end{array}$} & \multicolumn{3}{|c|}{ First group } & \multicolumn{2}{|c|}{ Second group } \\
\hline & & $\begin{array}{l}\text { Patients } \\
(n=399)\end{array}$ & $\begin{array}{l}\text { Controls } \\
(n=376)\end{array}$ & $\begin{array}{c}\text { Functional } \\
\text { validation }\end{array}$ & $\begin{array}{l}\text { Patients } \\
(n=383)\end{array}$ & $\begin{array}{l}\text { Controls } \\
(n=104)\end{array}$ \\
\hline \multicolumn{7}{|l|}{ N-terminal region } \\
\hline c.95T $>A$ & p.M32L & 5 & 2 & & & \\
\hline c. $403 \mathrm{~A}>\mathrm{G}$ & p.S135R & 1 & 1 & & & \\
\hline c. $953 \mathrm{C}>\mathrm{T}$ & p.A318V & 1 & 0 & & & \\
\hline c. $1088 \mathrm{G}>\mathrm{C}$ & p.R363T & 1 & 0 & & & \\
\hline c. $1315 \mathrm{G}>\mathrm{T}$ & p.E439x & 1 & 0 & & & \\
\hline c. $1753 \mathrm{C}>\mathrm{T}$ & p.P585S & 1 & 2 & & & \\
\hline \multicolumn{7}{|l|}{ C2-domain regions } \\
\hline c. $1767 \mathrm{G}>\mathrm{T}$ & p.Q589H & 4 & 2 & Non-impaired & & \\
\hline c. $1793 \mathrm{G}>\mathrm{A}$ & p.R598Q & 3 & 0 & Impaired & & \\
\hline c. $1808 \mathrm{G}>\mathrm{C}$ & p.C603S & & & & 1 & 0 \\
\hline c. $1904 C>G$ & p.A635G & 2 & 0 & Impaired & & \\
\hline c. $1913 \mathrm{C}>\mathrm{T}$ & p.T638I & & & & 1 & 0 \\
\hline c. $2291 \mathrm{C}>\mathrm{T}$ & p.A764V & 1 & 0 & Non-impaired & & \\
\hline c. $2417 \mathrm{C}>\mathrm{T}$ & p.T806I & 1 & 0 & Impaired & & \\
\hline c. $2435 \mathrm{G}>\mathrm{A}$ & p.R812H & 1 & 1 & Non-impaired & & \\
\hline c. $2441 \mathrm{G}>\mathrm{T}$ & p.R814 L & & & & 1 & 0 \\
\hline c. $2510 \mathrm{C}>\mathrm{G}$ & p.A837G & 2 & 0 & Impaired & & \\
\hline c. $2512 A>G$ & p.1838V & 2 & 0 & Impaired & & \\
\hline c. $2521 \mathrm{G}>\mathrm{A}$ & p.A841T & & & & 1 & 0 \\
\hline c. $2555 G>A$ & p.R852Q & & & & 4 & 2 \\
\hline c. $2648 \mathrm{G}>\mathrm{A}$ & p.G883D & & & & 1 & 0 \\
\hline Total variants* & & 26 & 8 & & NA & NA \\
\hline Variants C2-domains & & 16 & 3 & & 9 & 2 \\
\hline First + second group & & $n=782$ & $n=480$ & & & \\
\hline Total C2-domain** & & 25 & 5 & & & \\
\hline Variants with impaired & $\operatorname{up}^{* * *}$ & 10 & 0 & & & \\
\hline
\end{tabular}

Abbreviations: NA, not applicable; RPGRIP1, retinitis pigmentosa GTPase regulator-interacting protein 1.

${ }^{*} P<0.003$ (two-tailed Fischer's exact test), $\mathrm{OR}=2.8(95 \% \mathrm{Cl}=1.4-5.5)$ for identified total variants in first group.

$* * P<0.013, \mathrm{OR}=2.5(95 \% \mathrm{Cl}=1.2-5.3)$ for identified $\mathrm{C} 2$-domains total variants in first and second group.

$* * * P<0.001, \mathrm{OR}=7.1(95 \% \mathrm{Cl}=2.2-23.1)$ for identified $\mathrm{C} 2$-domains variants with impaired function in first group. 


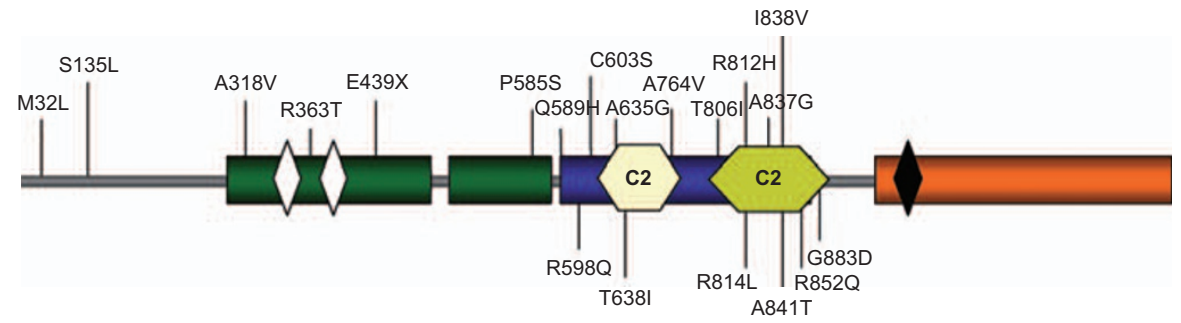

Figure 1 RPGRIP1 protein structure and location of amino acids changes identified in this study. Schematic representation of the protein domains (modified from Roepman et al ${ }^{21}$; GeneBank NP_065099) highlighting the location of the C2-domains region. All amino acids changes identified in the discovery group are listed upward; changes identified in the replication group are listed below the structure. Green bars represent coiled-coil domains, orange bar the RPGRinteracting domain, light yellow and green bar the C2 domains, white bars leucine zipper domains and dark bar the bipartite nuclear localization signal.

Table 2 Phenotypic characteristics of patients with RPGRIP1 mutations

\begin{tabular}{|c|c|c|c|c|c|c|c|c|}
\hline Subject ID & $\begin{array}{l}\text { RPGRIP1 } \\
\text { variants }\end{array}$ & $\begin{array}{c}\text { MYOC } \\
\text { variants }\end{array}$ & $\begin{array}{l}\text { CYP1B1 } \\
\text { variants }\end{array}$ & Phenotype & $\begin{array}{c}\text { Age at recruitment } \\
\text { (years) }\end{array}$ & $\begin{array}{c}\operatorname{Max} I O P \\
(m m H g) R / L\end{array}$ & $\begin{array}{c}\text { Optic } \\
\text { disk } R / L^{\mathrm{a}}\end{array}$ & $\begin{array}{l}\text { Disk area } \\
(\mathrm{mm} 2) \mathrm{R} / \mathrm{L}\end{array}$ \\
\hline \multicolumn{9}{|l|}{ First group } \\
\hline 99034 & M32L & - & - & POAG & 44 & $25 / 26$ & ND & ND \\
\hline 99440 & M32L & - & - & NTG & 60 & $21 / 21$ & ND & ND \\
\hline 13718 & M32L & - & - & POAG & 65 & $30 / 30$ & IV/V & $2.6 / 2.6$ \\
\hline 17163 & M32L & - & - & POAG & 72 & $40 / 40$ & IV/IV & $2.6 / 2.6$ \\
\hline 17483 & M32L & - & - & POAG & 73 & $27 / 27$ & $|/| I \mid$ & $2.1 / 2.6$ \\
\hline 7191 & S135R & - & - & POAG & 50 & $26 / 27$ & II/II & $3.7 / 3.6$ \\
\hline 19418 & $\mathrm{~A} 318 \mathrm{~V}$ & - & - & POAG & 70 & $31 / N D$ & III/ND & $2.6 / \mathrm{ND}$ \\
\hline 8562 & R363T & - & - & NTG & 44 & $21 / 21$ & $\mid I / I I$ & $3.3 / 3.7$ \\
\hline 99435 & E439X & - & - & NTG & 58 & $20 / 20$ & $\|/ /\|$ & $3.1 / 2.7$ \\
\hline 10554 & P585S & - & - & POAG & 63 & $26 / 26$ & $\|/ /\|$ & ND \\
\hline \multicolumn{9}{|c|}{ C2-domain regions } \\
\hline 10653 & Q589H & - & - & POAG & 69 & $21 / 24$ & III/IV & $1.6 / 2.1$ \\
\hline 13652 & $\mathrm{Q} 589 \mathrm{H}$ & - & - & POAG & 53 & $26 / 26$ & $\| / / I$ & $2.8 / 3.1$ \\
\hline 99168 & Q589H & Q368X & N203S & POAG & 49 & $38 / 38$ & $1 / 1$ & $2.0 / 1.9$ \\
\hline 21035 & Q589H & - & - & JOAG & 25 & $22 / 36$ & $0 / 4$ & $1.9 / 2.4$ \\
\hline 99192 & $\mathrm{R} 598 \mathrm{Q}$ & - & - & POAG & 43 & $24 / 24$ & ND & ND \\
\hline 99302 & $\mathrm{R} 598 \mathrm{Q}$ & - & - & POAG & 44 & $26 / 26$ & $\| I 1 / I \mid$ & ND \\
\hline 99242 & $\mathrm{R} 598 \mathrm{Q}$ & - & - & JOAG & 24 & $35 / 35$ & $1 / 1$ & $2.9 / 3$ \\
\hline 14501 & A635G & - & - & NTG & 68 & $21 / 21$ & $\mid \mathrm{I} / \mathrm{I}$ & ND \\
\hline 19243 & A635G & - & - & POAG & 81 & ND & ND & ND \\
\hline 13747 & A764V & - & - & POAG & 69 & $28 / 28$ & $\|/ /\|$ & $2.9 / 2.7$ \\
\hline 10033 & T806। & E352K & - & POAG & 49 & ND & ND & ND \\
\hline 10540 & $\mathrm{R} 812 \mathrm{H}$ & - & - & NTG & 66 & $20 / 20$ & ND & ND \\
\hline 16886 & A837G & - & - & POAG & 67 & ND & $\mathrm{V} / \mathrm{III}$ & $2.85 / 2.89$ \\
\hline 10366 & A837G & - & - & JOAG & 20 & $24 / 20$ & IV/IV & ND \\
\hline 11638 & $1838 \mathrm{~V}$ & - & - & NTG & 41 & $20 / 22$ & I/I & $4.5 / 4.3$ \\
\hline 21614 & $1838 \mathrm{~V}$ & - & - & POAG & 53 & $30 / 32$ & $1 / /$ & $2.8 / 2.8$ \\
\hline
\end{tabular}

Second group ${ }^{\mathrm{b}}$

C2-domain regions

\begin{tabular}{|c|c|c|c|c|c|c|c|c|}
\hline 154 & C603S & - & - & NTG & 50 & $10 / 10$ & $0 / 0$ & $2.4 / 2.4$ \\
\hline 210 & T638I & - & - & NTG & 65 & $17 / 16$ & $0 / 1$ & ND \\
\hline 58 & R814L & - & - & POAG & 59 & $26 / 26$ & $11 / 0$ & ND \\
\hline 290 & A841T & - & - & NTG & 29 & $23 / 23$ & $1 / 0$ & $0.7 / 0.7$ \\
\hline 24 & R852Q & - & - & NTG & 78 & $19 / 18$ & I/I & $0.7 / 0.7$ \\
\hline 47 & R852Q & - & - & NTG & 67 & $18 / 19$ & III/O & $2.4 / 2.1$ \\
\hline 148 & R852Q & - & - & NTG & 88 & $15 / 16$ & ND & $1.9 / 1.7$ \\
\hline 149 & R852Q & - & - & NTG & 83 & $20 / 18$ & $|/ I|$ & $2.6 / 2.6$ \\
\hline 292 & G883D & - & - & NTG & 37 & $16 / 16$ & ND & $0.5 / 0.3$ \\
\hline
\end{tabular}

Abbreviations: CYP1B1, cytochrome P450-1B; JOAG, juvenile open-angle glaucoma; Max IOP, maximum intraocular pressure at time of diagnosis (Goldmann's applanation tonometry); MYOC, myocilin; NRR, neuroretinal rim; NTG, normal tension glaucoma; POAG, primary open-angle glaucoma; RPGRIP1, retinitis pigmentosa GTPase regulator-interacting protein 1.

${ }^{a}$ Grade of optic disk atrophy according to Jonas and Papastathopoulos ${ }^{42}$ (from normal 0, moderate cupping I, notching of the NRR II-III, temporal NRR loss IV to complete atrophy V). bis. field: visual field in second group with Aulhorn. 

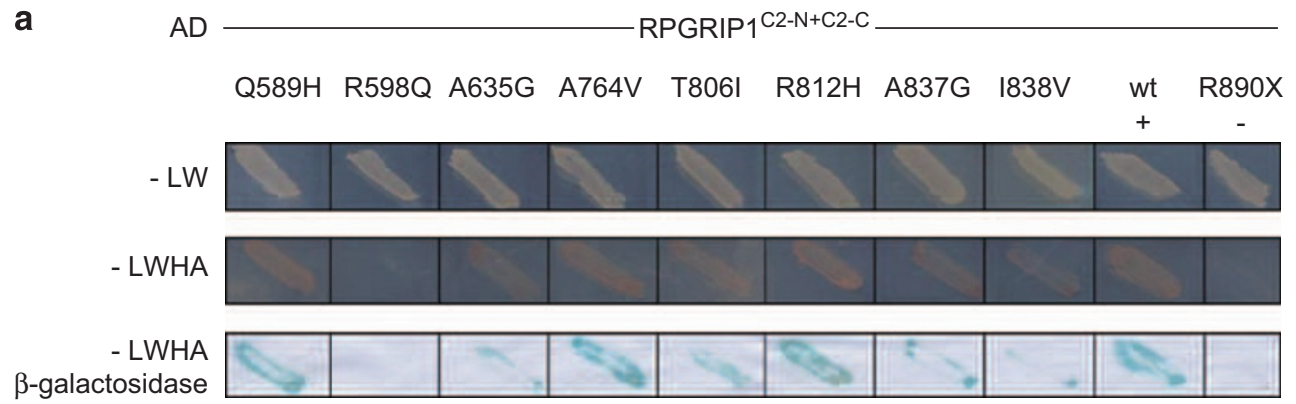

$\beta$-galactosidase

$\mathrm{BD}$

NPHP4f

b

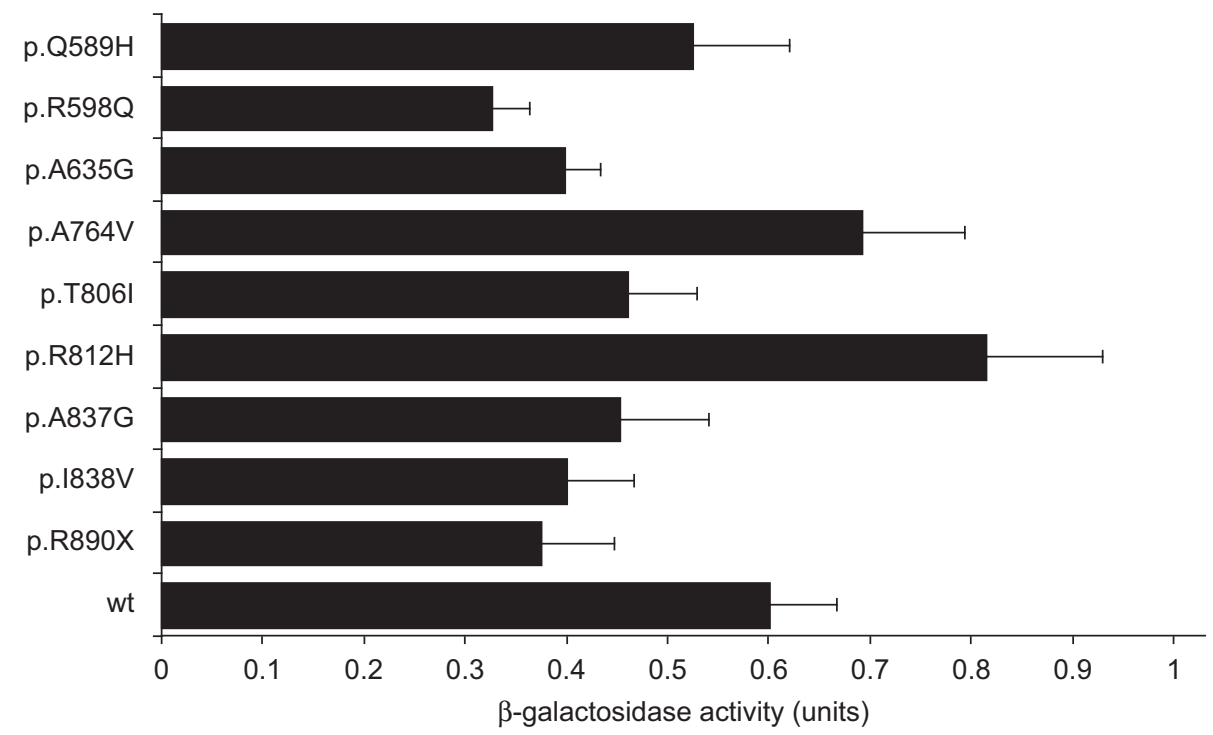

Figure 2 Effect of RPGRIP1 mutations on interaction with nephrocystin-4 (NPHP4). (a) Wild-type (wt) and mutated human RPGRIP1 2 -N+C2-C proteins (fused to GAL4 transcription activation domain) were assessed for interaction with NPHP4, fused to the GAL4-binding domain. As a negative control, the known loss of function mutation, p.R890X-RPGRIP1, was used. Media lacking the amino acids Leu and Trp was used to select for cotransformants (-LW panel). Additional omission of His and Ade from the media selected for activation of associated HIS3 and ADE2 reporter genes (-LWHA panel). Blue staining indicates $\beta$-galactosidase activity by activation of the LacZ reporter gene. (b) Results of liquid $\beta$-galactosidase assays. Black bars indicate the average enzymatic activity of each construct. The error bars show standard deviation. Both approaches show that binding with NPHP4 was severely disrupted

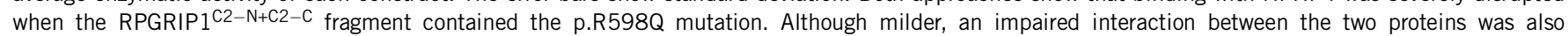
revealed by RPGRIP1 variants p.A635G, p.T806I, p.A837G and p.I838V. In contrast, RPGRIP1s p.Q589H, p.A764V and p.R812H did not cause any decrease in the interaction between RPGRIP1 and NPHP4, presenting similar or higher $\beta$-galactosidase activity to that of the wild-type protein.

found in glaucoma and healthy patients located within or around the C2 domains of RPGRIP1 (Table 1 and Figure 1), we evaluated the effect of 8 of the 15 variants on the interaction activity between RPGRIP1 and NPHP4 with yeast two-hybrid assay. As shown in Figure $2 \mathrm{a}$, reduced interaction activity compared with the wild-type control was observed qualitatively by $\beta$-galactosidase staining of yeast transformants with the RPGRIP1 constructs p.R598Q, p.A635G, p.T806I, p.A837G and p.I838V, whereas no changes were noted with the p.Q589H, p.A764V and p.R812H variants. These observation were validated with semiquantitative $\beta$-galactosidase assays of yeast transformants harboring identical constructs (Figure 2b), although the p. $\mathrm{R} 812 \mathrm{H}$ construct had a higher binding activity than the wild-type protein. These results support that the variants p.A635G, p.T806I, p.A837G and p.I838V, which are found only in disease patients and within the C2 domains of RPGRIP1, impair the interaction of the C2 domains of RPGRIP1 with NPHP4, whereas the p.R598Q also found only in glaucoma patients may have an indirect (conformational) effect over the C2 domains of RPGRIP1 similarly to that observed with the truncation p. R890X (negative control). Finally, independent validation of the impairment of the interaction between the p.R598Q variant and NPHP4 was confirmed by coimmunoprecipitation assays of extracts of cells co-transfected with NPHP4 and wild-type or p.R598Q mutant constructs of the C2 domains of RPGRIP1 (Figure 3).

Taking into account only the variants of the first group with impaired RPGRIP1-NPHP4 interaction (p.R598Q, p.A635G, p.T806I, p.A837G and p.I838V) we could count a total of five variants in10 patients $(2.8 \%)$ and none in healthy subjects (Table 1$)$. This resulted in a significant increase of the effect over expectancy $(P=0.001$, two-tailed Fischer's exact test; $\mathrm{OR}=7.1,95 \% \mathrm{CI}=2.2-23.1$, Table 1).

\section{DISCUSSION}

Our results suggest an implication of the RPGRIP1 gene as susceptibility gene in various forms of glaucoma.

Through alternative splicing, the RPGRIP1 gene expresses several isoforms in ocular tissues. ${ }^{22,23}$ These isoforms have different cellular and subcellular locations, biochemical properties, and species-specific expression among retinal neuron and also outside retina. ${ }^{23-26}$ 


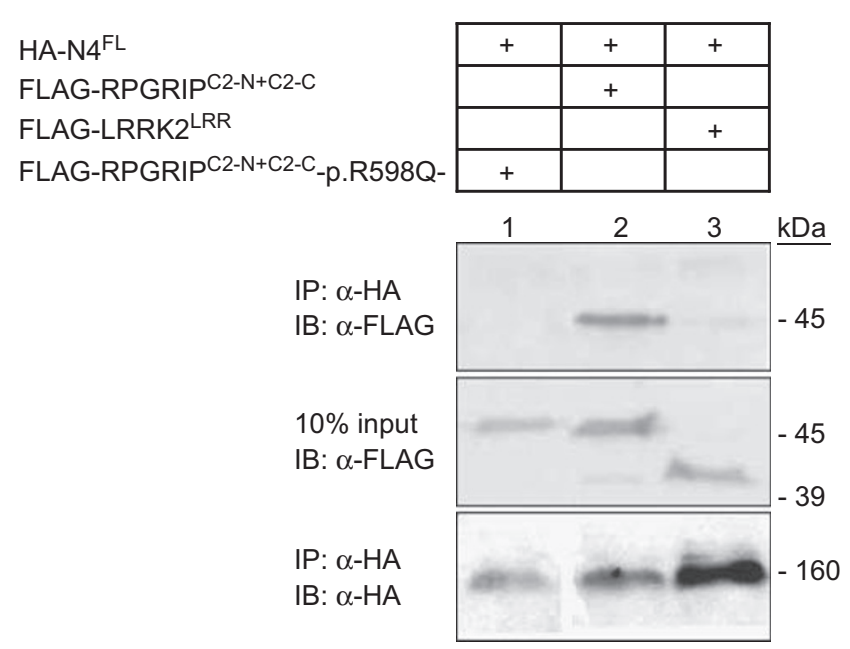

Figure 3 Coimmunoprecipitation of nephrocystin-4 (NPHP4) and RPGRIP1 p.R598Q mutation. The top immunoblot indicates specific coimmunoprecipitation of full-length NPHP4 and RPGRIP1 fragments containing both C2 domains of the protein (lane 2). The RPGRIP1 construct containing the p.R598Q alteration was not found to coimmunoprecipitate with NPHP4 ${ }^{\mathrm{FL}}$ (lane 1), suggesting that the interaction between both proteins was severely disrupted. As a negative control, LRRK2 ${ }^{\mathrm{LRR}}$ did not coimmunoprecipitate with NPHP4 ${ }^{\mathrm{FL}}$ (lane 3 ). Protein inputs $(10 \%)$ are shown in the middle blot. The bottom immunoblot shows immunoprecipitation of HA-tagged NPHP4FL with anti-HA beads. The sizes $(\mathrm{kDa})$ of the proteins corresponding with the specific antibody signals are indicated.

The RPGRIP1 protein contains three structurally different regions. An $\mathrm{N}$-terminal coiled-coil domains with homology to SMC proteins, ${ }^{24,27}$ which possibly mediates homotypic and/or heterotypic interactions; ${ }^{27}$ a C-terminal region that contains the RPGR-interacting domain (RID); ${ }^{23}$ and a central region that contains a protein kinase $\mathrm{C}$ conserved region $2(\mathrm{C} 2)$ motif mediating protein-protein interactions. ${ }^{28,29}$ A known interacting partner of the C2 domains is NPHP4. Mutations located in RPGRIP1 C2 domain disrupt the RPGRIP1NPHP4 complex and cause Leber congenital amaurosis (LCA). ${ }^{21}$ Another known interaction partner of the RPGRIP1 C-terminal region (RID) is the $\mathrm{X}$-linked $R P G R$ gene, involved in $\mathrm{X}$-linked retinitis pigmentosa type $3 .^{22}$ Furthermore, RPGRIP1 has been detected in the retina as a complex with the basal body protein CEP290 30 and in amacrine cells with the neuronal nucleoporin RANBP2. ${ }^{24}$ These results suggest that one of the main roles of one or more RPGRIP1 isoforms is to serve as a plastic and dynamic scaffold for proteins or protein modules acting in signaling pathways of different retinal cell subpopulations, thus linking one or more RPGRIP1 isoforms to a set of different clinical phenotypes. ${ }^{31,32}$

After analysis of the 10 candidate genes as reported in the results section, we sequenced the entire RPGRIP1 coding region in a large cohort of POAG patients as we expected to find association to rare variants rather than common polymorphisms (SNPs), in line with previous findings in other glaucoma genes, such as MYOC, ${ }^{33}$ WDR $36,{ }^{19}$ CYP $1 B 11^{13}$ and NTF4 ${ }^{14}$ In addition, previous data support the hypothesis that POAG is characterized by a high locus and allelic heterogeneity with different rare variants in numerous genes. ${ }^{7}$ Most rare variants are likely to be missense variants, whose functional effects may be expected to arise mainly from amino acid changes that affect protein-protein interactions and that can thus have mildly dominant or dominant-negative effects. Under these considerations, many variants with small effects, a smaller number with intermediate effects and relatively few with large effects are then expected to be found. ${ }^{34}$

Indeed we identified several different missense variants in RPGRIP1 in almost $6.5 \%$ of cases, $4 \%$ of these are located in the C2-domains of the protein $(P=0.004)$. When we sequenced the second cohort of German patients, we focused our attention on variants located in the structurally and functionally important $\mathrm{C} 2$-domain regions. Also in this second cohort we detected different missense variants located in the $\mathrm{C} 2$-domains but at a lower frequency rate (2.3\%). We hypothesize that this could be because of possible different characteristics of the second cohort composed mainly of NTG patients.

To determine whether the eight $\mathrm{C} 2$-domains variants identified in the first group are essential for the structure of the C2-domains, we performed yeast two-hybrid experiments using NPHP4, the only currently known binding partner. ${ }^{21}$ Five of them indeed showed impaired interaction (Table 1), suggesting that the variants are functionally relevant and not benign polymorphisms. When taking into account only these five variants (p.R598Q, p.A635G, p.T806I, p.A837G and p.I838V) detected in 10 patients and in none of the control subjects, the association remained significant $(P=0.001$, Table 1). As RPGRIP1 encodes scaffold protein isoforms, ${ }^{22}$ additional binding partners are likely to exist in the retina or tissues etiologically relevant to glaucoma (eg, trabecular meshwork), and therefore we hypothesize that the other variants identified might affect the composition of RPGRIP1 complexes that might be relevant to the pathogenesis of glaucoma.

The detected effect of the C2-domain variants in protein-protein interaction, however, is mostly not pronounced and it might be compensated in cell sub-populations for many years. This is in line with glaucoma being a late on set disease with complex inheritance. In fact, age or other risk factors, such as elevated IOP, might be required to manifest the disease.

Similar to other glaucoma genes described to this date, the mutations found in RPGRIP1 are monoallelic. This is in contrast to all homozygous or compound heterozygous mutations in RPGRIP1 that are linked to inherited photoreceptor dystrophies, such as LCA. ${ }^{35,36}$ Interestingly, the presence of clinical manifestations has also been reported in heterozygous carriers of LCA associated RPGRIP1 mutations characterized by reduced dark/light-ERG responses and abnormal ERG amplitudes. ${ }^{37}$ Similarly, delayed ERG responses and reduced amplitudes have been also shown in glaucoma. ${ }^{38}$

Our clinical and genetic data support the hypothesis that retinal ganglion cell axon degeneration and loss of retina ganglion cells in POAG is not only due to elevated IOP alone but due to other concomitant factors compromising the survival of neuronal cell subpopulations in the inner retina as we recently reported. ${ }^{14}$ Therefore, the putative role of RPGRIP1 as a scaffold protein regulating different signal pathways among different neuronal cell populations of the retina and its association with glaucoma makes RPGRIP1 an interesting functional candidate gene to dissect pathobiological processes underlying POAG, JOAG and NTG.

Interestingly, two patients, one with POAG and one with JOAG, carry also mutations in MYOC and CYP1B1 (Table 2). To date a digenic inheritance in open-angle glaucoma has been reported in patients with mutations in both MYOC and CYP1B1. ${ }^{13,39}$ Thus, on the basis of present knowledge, it is likely that the POAG in patient ID99168 (Table 2) is mainly due to the presence of mutations p.Q368X in MYOC and p.N203S in CYP1B1, as these have been previously confirmed to have a pathological function. ${ }^{13,40}$ In fact, according to our experimental results, the p.Q589H in RPGRIP1 seems to be non-pathological (Table 1). In patient ID10033 
(Table 2) instead, the JOAG phenotype may be due mainly to the pathological p.T806I mutation in RPGRIP1 (Table 1) as the MYOC mutation, p.Q352K, has been previously reported to be a probable benign sequence change. ${ }^{41}$ Alternatively, it is possible that in patient ID10033 there is epistatic interaction between p.T806I mutation in RPGRIP1and the MYOC mutation, p.Q352K, because of the mild impairment effect of p.T806I mutation on the interaction of the $\mathrm{C} 2$ domains of RPGRIP1 with NPHP4.

Though, our study has some limitations, we focused our attention mainly on genes expressed in retina considering the process of retinal ganglion cell degeneration as a common etiological denominator for these glaucoma forms. However, we cannot exclude from the chromosomal region analyzed the presence of other candidate genes expressed in other tissues that might have a critical role in the pathogenesis of glaucoma, such as trabecular meshwork or ciliary body. We also could not prove segregation or de novo occurrence of these variants as no family members were available for study, a situation not uncommon for late onset diseases. Finally, the power of our study is limited and will require independent validation.

In summary, our data suggest that rare heterozygous loss of function variants in RPGRIP1 are a risk factor for POAG and other forms of glaucoma, such as NTG and JOAG, and support the hypothesis that genetic predisposition to this disease is mainly caused by rare non-synonymous variants rather than common SNPs.

\section{CONFLICT OF INTEREST}

The authors declare no conflict of interest.

\section{ACKNOWLEDGEMENTS}

We thank all patients and control individuals for their participation, Olga Zwenger for technical assistance and Juliane Niedzella for patients' recruitment support. This study was supported by Grant SFB 539 from the German Research Foundation. PAF was supported by grants from the National Institutes of Health (GM083165, EY019492 and 2P30-EY005722). PAF is the Jules and Doris Stein Research to Prevent Blindness Professor.

\section{WEB RESOURCES}

The URLs for data presented herein are as follows:

National Center for Biotechnology Information (NCBI), http://www.ncbi.nlm. nih.gov/

Genome Browser of the University of California Santa Cruz (UCSC), http:// genome.ucsc.edu/cgi-bin/hgTracks (reference sequences ssed: NT_026437 and NM_020366 and NP_065099)

Ensembl Database (http://www.ensembl.org).

ClustalW, http://www.ebi.ac.uk/clustalw/

Online Mendelian Inheritance in Man (OMIM): http://www.ncbi.nlm.nih.gov/ entrez/query.fcgi? $\mathrm{db}=\mathrm{OMIM} /$.

1 Kwon YH, Fingert JH, Kuehn MH, Alward WL: Primary open-angle glaucoma. N Eng/ J Med 2009; 360: 1113-1124.

2 Thylefors B, Negrel AD, Pararajasegaram R, Dadzie KY: Global data on blindness. Bull World Health Organ 1995; 73: 115-121.

3 Quigley HA, Broman AT: The number of people with glaucoma worldwide in 2010 and 2020. Br J Ophthalmol 2006; 90: 262-267.

4 Shields MB: Staging glaucoma for the twenty-first century: sixth Chandler-Grant Lecture. J Glaucoma 1996; 5: 340-344.

5 Wiggs JL, Damji KF, Haines JL, Pericak-Vance MA, Allingham RR: The distinction between juvenile and adult-onset primary open-angle glaucoma. Am J Hum Genet 1996; 58: 243-244

6 Wiggs JL: Genetic etiologies of glaucoma. Arch Ophthalmol 2007; 125: 30-37.

7 Allingham RR, Liu Y, Rhee DJ: The genetics of primary open-angle glaucoma: a review. Exp Eye Res 2009; 88: 837-844.

8 Stone EM, Fingert JH, Alward WL et al: Identification of a gene that causes primary open angle glaucoma. Science 1997; 275: 668-670.
9 Rezaie T, Child A, Hitchings R et al: Adult-onset primary open-angle glaucoma caused by mutations in optineurin. Science 2002; 295: 1077-1079.

10 Monemi S, Spaeth G, DaSilva A et al: Identification of a novel adult-onset primary open-angle glaucoma (POAG) gene on 5q22.1. Hum Mol Genet 2005; 14: 725-733.

11 Fan BJ, Wang DY, Lam DS, Pang CP: Gene mapping for primary open angle glaucoma. Clin Biochem 2006; 39: 249-258.

12 Fuse N: Genetic bases for glaucoma. Tohoku J Exp Med 2010; 221: 1-10.

13 Pasutto F, Chavarria-Soley G, Mardin CY et al: Heterozygous loss of function variants in CYP1B1 predispose to primary open angle glaucoma. Invest Ophthalmol Vis Sci 2010; 51: 249-254.

14 Pasutto F, Matsumoto T, Mardin CY et al: Heterozygous NTF4 mutations impairing neurotrophin-4 signaling in patients with primary open-angle glaucoma. Am J Hum Genet 2009; 85: 447-456.

15 van Koolwijk LM, Despriet DD, van Duijn CM et al: Genetic contributions to glaucoma: heritability of intraocular pressure, retinal nerve fiber layer thickness, and optic disc morphology. Invest Ophthalmol Vis Sci 2007; 48: 3669-3676.

16 Berdahl JP, Allingham RR, Johnson $\mathrm{DH}$ : Cerebrospinal fluid pressure is decreased in primary open-angle glaucoma. Ophthalmology 2008; 115: 763-768.

17 Wiggs JL, Allingham RR, Hossain A et al: Genome-wide scan for adult onset primary open angle glaucoma. Hum Mol Genet 2000; 9: 1109-1117.

18 Nemesure B, Jiao X, He Q et al: A genome-wide scan for primary open-angle glaucoma (POAG): the Barbados Family Study of open-angle glaucoma. Hum Genet 2003; 112: 600-609.

19 Pasutto F, Mardin CY, Michels-Rautenstrauss K et al: Profiling of WDR36 missense variants in German patients with glaucoma. Invest Ophthalmol Vis Sci 2008; 49: 270-274.

20 Weisschuh N, Wolf C, Wissinger B, Gramer E: Variations in the WDR36 gene in German patients with normal tension glaucoma. Mol Vis 2007; 13: 724-729.

21 Roepman R, Letteboer SJ, Arts HH et al: Interaction of nephrocystin-4 and RPGRIP1 is disrupted by nephronophthisis or Leber congenital amaurosis-associated mutations. Proc Natl Acad Sci USA 2005; 102: 18520-18525.

22 Ferreira PA: Insights into X-linked retinitis pigmentosa type 3, allied diseases and underlying pathomechanisms. Hum Mol Genet 2005; 14 Spec No.2: R259-R267.

23 Roepman R, Bernoud-Hubac N, Schick DE et al: The retinitis pigmentosa GTPase regulator (RPGR) interacts with novel transport-like proteins in the outer segments of rod photoreceptors. Hum Mol Genet 2000; 9: 2095-2105.

24 Castagnet P, Mavlyutov T, Cai Y, Zhong F, Ferreira P: RPGRIP1s with distinct neuronal localization and biochemical properties associate selectively with RanBP2 in amacrine neurons. Hum Mol Genet 2003; 12: 1847-1863.

25 Lu X, Ferreira PA: Identification of novel murine- and human-specific RPGRIP1 splice variants with distinct expression profiles and subcellular localization. Invest Ophthalmol Vis Sci 2005; 46: 1882-1890.

26 Mavlyutov TA, Zhao H, Ferreira PA: Species-specific subcellular localization of RPGR and RPGRIP isoforms: implications for the phenotypic variability of congenital retinopathies among species. Hum Mol Genet 2002; 11: 1899-1907.

27 Lu X, Guruju M, Oswald J, Ferreira PA: Limited proteolysis differentially modulates the stability and subcellular localization of domains of RPGRIP1 that are distinctly affected by mutations in Leber's congenital amaurosis. Hum Mol Genet 2005; 14: 1327-1340.

28 Rizo J, Sudhof TC: C2-domains, structure and function of a universal Ca2+-binding domain. J Biol Chem 1998; 273: 15879-15882.

29 Nalefski EA, Falke JJ: The C2 domain calcium-binding motif: structural and functional diversity. Protein Sci 1996; 5: 2375-2390.

30 Chang $\mathrm{B}$, Khanna $\mathrm{H}$, Hawes $\mathrm{N}$ et al: In-frame deletion in a novel centrosomal/ciliary protein CEP290/NPHP6 perturbs its interaction with RPGR and results in early-onset retinal degeneration in the rd16 mouse. Hum Mol Genet 2006; 15: 1847-1857.

31 Arts HH, Cremers FP, Knoers NV, Roepman R: Focus on molecules: RPGRIP1. Exp Eye Res 2009; 88: 332-333.

32 Oti M, Huynen MA, Brunner HG: Phenome connections. Trends Genet 2008; 24: $103-106$.

33 Gong G, Kosoko-Lasaki O, Haynatzki GR, Wilson MR: Genetic dissection of myocilin glaucoma. Hum Mol Genet 2004; 13 Spec No 1: R91-R102.

34 Bodmer W, Bonilla C: Common and rare variants in multifactorial susceptibility to common diseases. Nat Genet 2008; 40: 695-701.

35 Dryja TP, Adams SM, Grimsby JL et al: Null RPGRIP1 alleles in patients with Leber congenital amaurosis. Am J Hum Genet 2001; 68: 1295-1298.

36 Booij JC, Florijn RJ, ten Brink JB et al: Identification of mutations in the AIPL1, CRB1, GUCY2D, RPE65, and RPGRIP1 genes in patients with juvenile retinitis pigmentosa. J Med Genet 2005; 42: e67.

37 Galvin JA, Fishman GA, Stone EM, Koenekoop RK: Clinical phenotypes in carriers of Leber congenital amaurosis mutations. Ophthalmology 2005; 112: 349-356.

38 Raz D, Perlman I, Percicot CL, Lambrou GN, Ofri R: Functional damage to inner and outer retinal cells in experimental glaucoma. Invest Ophthalmol Vis Sci 2003; 44: 3675-3684.

39 Vincent AL, Billingsley G, Buys $Y$ et al: Digenic inheritance of early-onset glaucoma: CYP1B1, a potential modifier gene. Am J Hum Genet 2002; 70: 448-460.

40 Aroca-Aguilar JD, Sanchez-Sanchez F, Martinez-Redondo F, Coca-Prados M, Escribano J: Heterozygous expression of myocilin glaucoma mutants increases secretion of the mutant forms and reduces extracellular processed myocilin. Mol Vis 2008; 14: 2097-2108.

41 Zhou Z, Vollrath D: A cellular assay distinguishes normal and mutant TIGR/myocilin protein. Hum Mol Genet 1999; 8: 2221-2228.

42 Jonas JB, Papastathopoulos K: Ophthalmoscopic measurement of the optic disc. Ophthalmology 1995; 102: 1102-1106. 